\title{
EL ENTRENAMIENTO EN ESTRATEGIAS SOBRE LA COMPRENSIÓN LECTORA DEL ENUNCIADO DEL PROBLEMA ARITMÉTICO: UN ESTUDIO EMPÍRICO CON ESTUDIANTES DE EDUCACIÓN PRIMARIA
}

\author{
TRAINING STRATEGIES IN RESPECT TO READING COMPREHENSION \\ BASED ON THE FORMULATION OF ARITHMETICAL PROBLEMS: \\ EMPIRICAL STUDY WITH PRIMARY SCHOOL PUPILS
}

\author{
Sonia Beltrán Campos* y Elvira Repetto Talavera** \\ Universidad Nacional de Educación a Distancia
}

\begin{abstract}
RESUMEN
En el proceso de resolución de problemas aritméticos una fase necesaria es la comprensión del enunciado y, más en concreto, la comprensión lectora del enunciado. Este requisito permite al resolutor extraer un modelo mental de las relaciones que se extraen de las premisas del enunciado, permitiéndole de este modo focalizar su atención en la comprensión matemática del mismo. El artículo presenta los resultados obtenidos de una investigación llevada a cabo en la Comunidad de Madrid poniéndose de manifiesto el beneficio obtenido en la comprensión lectora del enunciado, junto a otras variables relacionadas con el proceso de resolución de problemas, tras haber sometido a un grupo de estudiantes de Educación Primaria a un entrenamiento en estrategias comprensivas y metacomprensivas.
\end{abstract}

Palabras clave: resolución de problemas, comprensión lectora del enunciado verbal del problema aritmético, entrenamiento es estrategias comprensivas y metacomprensivas.

\section{ABSTRACT}

One prerequisite regarding the resolution of arithmetical problems, in this case, for primary school pupils, is the reading and comprehension of the formulation. This enables the solver to construct a mental model of the meaning of the problem formulation, leading him/her to focus his/her attention

\footnotetext{
* Profesora Ayudante en el Departamento MIDE II (Orientación Educativa, Diagnóstico e Intervención Psicopedagógica) en la Facultad de Educación de la U.N.E.D. Madrid. sbeltran@edu.uned.es.

** Catedrática de Orientación Educativa. en el Departamento MIDE II (Orientación Educativa, Diagnóstico e Intervención Psicopedagógica) en la Facultad de Educación de la U.N.E.D. erepetto@edu.uned.es.
} 
towards resolving the problem. In this article, we present the results of our research, carried out within Comunidad de Madrid. These results highlight the importance of reading and comprehension, along with other skills needed for the resolution of arithmetical problems. This conclusion was reached following a training exercise aimed at primary school pupils, in the area of comprehensive and metacomprehensive strategies.

Key words: Problem resolution, reading comprehension of a verbal arithmetical problem, training in comprehensive and metacomprehensive strategies.

\section{Marco teórico}

El concepto de Comprensión Lectora del Problema Aritmético de Enunciado Verbal aúna en sí dos áreas de conocimiento, por una parte, la comprensión lectora -área del lenguaje - y, por otra, la resolución de problemas —área de las matemáticas-. El Problema Aritmético de Enunciado Verbal de ahora en adelante P.A.E.V. (Castro, 1995) es una descripción verbal de una situación problemática en la que existen una o más cuestiones que resolver por medio de la aplicación del operaciones aritméticas. Si el soporte empleado es el texto escrito (Chamorro, 2003), en consecuencia, para determinar qué operaciones aritméticas son las necesarias para resolver el problema es preciso que se produzca un proceso de razonamiento. Sin embargo, cuando el alumno es capaz de resolver un problema sin la necesidad de comprenderlo estaría desarrollando lo que se denomina una "Transcripción Simbólica Aritmética" (TSA) (Fernández Bravo, 2000: 14), una asociación directa entre la situación expresada en el enunciado de un problema con una operación aritmética, por lo cual, el resolutor no se encontraría ante una verdadera situación de problema.

La comprensión del enunciado se señala como un requisito para acceder a la resolución de los problemas aritméticos (Anderson, 1993; Barnsford y Stein, 1993; Mason, Burton y Stacey, 1989; Mayer, 1986a, 1986b, 2003; Maza, 1995; Pérez Echeverría, 1993; Polya, 1990; Verschaffel, Greer y De Corte, 2000). Sin embargo, fueron Kintsch y Greeno (1985) quienes, basándose en las aportaciones de la teoría proposicional de Van Dijk y Kintsch (1983), establecen por primera vez un modelo de resolución de problemas verbales aritméticos en el que se incluye la relevancia del papel de la comprensión lectora. Los componentes más importantes de este modelo son: la representación del enunciado, esto es, la construcción de un "modelo de situación", las estrategias de comprensión lectora y los esquemas de conocimiento. A partir de este modelo, durante la primera fase de comprensión del enunciado del problema se debe diferenciar entre: la comprensión lectora del enunciado verbal y la comprensión matemática del enunciado del problema, en función del tipo de conocimiento requerido (no matemático y matemático, respectivamente).

Entre ambas formas de comprensión se puede establecer un paralelismo entre las fases de comprensión del problema de Mayer (1986a, 1986b; 2003), y la representación que obtiene el lector en función del conocimiento activado siguiendo la terminología de Kintsch y Greeno. Mientras la sub-fase de traducción hace referencia al texto-base del enunciado, es decir, la representación de la información contenida en el enunciado de forma descriptiva (detección de ideas, datos o incógnita), la sub-fase de interpretación añade a la anterior la representación de los diferentes tipos de conocimiento que posee el resolutor, entre ellos, el matemático, generando el modelo de situación. Si esto no sucediera, el modelo de 
situación generado por el resolutor se basará sólo en los aspectos léxicos, sintácticos y semánticos del enunciado y no constituirá una representación matemática de la situación problema.

Entre las diferentes variables que se relacionan con una actuación desigual entre los buenos y malos resolutores de problemas merecen destacarse las siguientes: el empleo desigual de la memoria operativa y a largo plazo (Geary y Orad, 2003); la cantidad y calidad de los conocimientos previos (Carr y Hettinger, 2003; Pérez Echeverría y Pozo, 1994) o la escasa activación de los conocimientos previos acerca del mundo real (Maza, 1995), los alumnos expertos emplean un mayor número de estrategias metacognitivas y de comprensión lectora en resolución de problemas (Anderson, 1984; Chamorro, 2003; Kinstch y Greeno, 1985; Miranda Fortes y Gil, 2000; Pérez Echevarría, 1994; Pozo y Postigo, 1993; Schoenfeld, 1985, 1992). Las estrategias comprensivas y metacomprensivas permiten al alumno enfocar la lectura de forma profunda y, en consecuencia, a no operar de forma prematura con los datos del enunciado como resultado de una lectura superficial. Si las estrategias comprensivas, así como el conocimiento y la regulación de dicha actividad, favorece la comprensión lectora, es obvio que también favorecerá la comprensión lectora en los enunciados de los problemas aritméticos. En esta investigación se pretende contrastar el beneficio del entrenamiento en estrategias comprensivas y metacomprensivas, seleccionadas a partir de algunas unidades del Programa Comprender y Aprender en el Aula (Repetto y cols; 2001), en la comprensión lectora del enunciado junto con otras variables relacionadas con el proceso de resolución de problemas.

\section{Objetivo del estudio}

El objetivo de la investigación reside en averiguar la incidencia que el entrenamiento en estrategias de comprensión y metacomprensión lectora produce en la Comprensión Lectora del Enunciado Verbal del Problema Aritmético, así como en otras variables relacionadas, en estudiantes de segundo y tercer ciclo de Educación Primaria de la Comunidad de Madrid.

\section{Diseño}

Para su consecución, la investigación parte de un diseño cuasi-experimental. En concreto, dentro de los diseños de dos grupos no equivalentes, se opta por un diseño con grupo control no equivalente con medidas pre y postratamiento (Ballester, 2001). Según este diseño, los grupos que se forman en base a la información que se recoge son los siguientes:

TABLA 1: Esquema de los grupos que se forman y de la información recogida.

\begin{tabular}{|l|c|c|}
\hline & Pretratamiento & Postratamiento \\
\hline Grupo experimental (G.E.) & Experimental-pretest & Experimental-postest \\
\hline Grupo control (G.C.) & Control-pretest & Control-postest \\
\hline
\end{tabular}




\section{Variables e instrumentos}

El tratamiento o la variable independiente consiste en la aplicación de algunas unidades seleccionadas del Programa Comprender y Aprender en el Aula, mediante las cuales, y a través de la mediación de profesor, se entrena a los alumnos en estrategias cognitivas y metacognitivas de comprensión lectora con objeto de que lleguen a ser eficaces y autónomos en su propio proceso lector. El entrenamiento y la ausencia del mismo en el G.E. y el G.C., respectivamente, es el criterio principal para diferenciar a ambos grupos. Las variable dependiente principal de nuestro estudio es la Comprensión Lectora del Enunciado Verbal del Problema Aritmético junto con otras como: la Comprensión Lectora el Cálculo, la Resolución de Problemas Aritméticos, el Razonamiento y la Memoria.

Los instrumentos de medida empleados son:

a) La batería psicopedagógica EVALÚA, de J. García Vidal y D. González Manjón (1996), publicada en la editorial EOS, está integrada por un conjunto de pruebas que tratan de evaluar la capacidad de aprendizaje de los alumnos de entre los 8 y 12 años de edad. Se divide en los siguientes niveles: 2 ( $3^{\circ}$ E.P.); 3 ( $4^{\circ}$ y $5^{\circ}$ E.P.) y $6\left(6^{\circ}\right.$ E.P.). La batería en total está formada por 15 pruebas, y evalúa el RZ, la M, el CA y la RP:

b) Las pruebas ACL de evaluación de la comprensión lectora $\left(1^{\circ}-6^{\circ}\right.$ de primaria) de $\mathrm{G}$. Catalá, M. Catalá, E. Molina y R. Monclús (2001). Se dirigen a valorar la Comprensión Lectora (CL) de una manera amplia, a partir de textos de diversa tipología y con temáticas referidas a las diferentes áreas del currículo (excepto en matemáticas o en resolución de problemas); $y$,

c) El Cuestionario de Comprensión Lectora del Enunciado Verbal del Problema Aritmético (CLEVPA) es un cuestionario elaborado ad hoc con el objetivo de identificar qué grado de comprensión lectora obtiene un alumno durante esta fase de resolución del problema en función de la aplicación de estrategias comprensivas y metacomprensivas. Comprende dos niveles: I ( $3^{\circ}$ y $4^{\circ}$ E.P. $)$ y II $\left(5^{\circ} \text { y } 6^{\circ} \text { E.P. }\right)^{1}$.

\section{Hipótesis}

Los alumnos del G.E. obtienen resultados significativamente mejores que los del G.C. en cada una de las siguientes variables: Comprensión Lectora (C.L.), Comprensión Lectora del Enunciado Verbal del Problema Aritmético (C.L.E.V.P.A.), Resolución de Problemas (R.P.), Razonamiento (R.Z.), Cálculo (CA) y Memoria (M) después del entrenamiento.

\section{Población y muestra}

La población a la que pertenece la muestra del estudio se extiende a los alumnos de segundo y tercer ciclo de Educación Primaria de la Comunidad de Madrid durante el curso es-

\footnotetext{
${ }^{3}$ Los análisis de validez e índices de fiabilidad están publicados en la Tesis Doctoral de S. Beltrán titulada: $L a$ comprensión lectora del problema aritmético: un entrenamiento con alumnos de Educación Primaria" (2005) en la U.N.E.D., aunque serán objeto de posteriores publicaciones científicas.
} 
colar 2001-2002. El proceso de muestreo llevado a cabo es intencional. La muestra se divide en 388 alumnos pertenecientes al G.E. y 200 alumnos al G.C.

\section{Análisis y resultados}

Antes de contrastar el posible beneficio producido tras el entrenamiento en estrategias entre el GE y el GC, es preciso comparar ambos grupos antes del tratamiento con objeto de garantizar la homogeneidad de la muestra. Con este fin, se realiza una prueba t para dos muestras independientes con la globalidad de ambas muestras:

TABLA 2: Diferencias de medias en las distintas variables antes del tratamiento en función del Grupo.

\begin{tabular}{|c|c|c|c|c|c|c|c|}
\hline Variables & \multicolumn{2}{|c|}{ Grupo } & Media & $\begin{array}{l}\text { Desv. } \\
\text { Tipic. }\end{array}$ & $\mathbf{T}$ & $\begin{array}{c}\text { Signf. } \\
\text { (bil) }\end{array}$ & Nivel \\
\hline \multirow{2}{*}{ Comprensión Lectora (CL) } & Exp. & $\mathrm{N}=337$ & 16,55 & 5,62 & \multirow{2}{*}{$-1,65$} & \multirow{2}{*}{, 09} & \\
\hline & Cont. & $\mathrm{N}=182$ & 17,42 & 5,77 & & & \\
\hline \multirow{2}{*}{ R. Problema 2 (RP 2) } & Exp. & $\mathrm{N}=49$ & 10,98 & 2,61 & \multirow{2}{*}{2,53} & \multirow{2}{*}{,26 } & \\
\hline & Cont. & $\mathrm{N}=50$ & 10,28 & 3,48 & & & \\
\hline \multirow{2}{*}{ Cálculo 2 (CA 2) } & Exp. & $\mathrm{N}=49$ & 47,08 & 8,86 & \multirow{2}{*}{,- 09} & \multirow{2}{*}{,92 } & \\
\hline & Cont. & $\mathrm{N}=51$ & 47,24 & 7,60 & & & \\
\hline \multirow{2}{*}{ Razonamiento 2 (RZ 2) } & Exp. & $\mathrm{N}=49$ & 76,08 & 16,03 & \multirow{2}{*}{,68 } & \multirow{2}{*}{,49 } & \\
\hline & Cont. & $\mathrm{N}=52$ & 74,02 & 14,24 & & & \\
\hline \multirow{2}{*}{ Memoria 2 (M2) } & Exp. & $\mathrm{N}=48$ & 76,71 & 18,84 & \multirow{2}{*}{1,12} & \multirow{2}{*}{, 12} & \\
\hline & Cont. & $\mathrm{N}=52$ & 70,94 & 18,33 & & & \\
\hline \multirow{2}{*}{ R. Problema 4 (RP 4) } & Exp. & $\mathrm{N}=248$ & 7,82 & 5,80 & \multirow{2}{*}{$-4,36$} & \multirow{2}{*}{, 00} & \multirow{2}{*}{$* *$} \\
\hline & Cont. & $\mathrm{N}=115$ & 10,75 & 6,23 & & & \\
\hline \multirow{2}{*}{ Cálculo 4 (CA 4) } & Exp. & $\mathrm{N}=248$ & 27,42 & 7,25 & \multirow{2}{*}{$-1,57$} & \multirow{2}{*}{, 00} & \multirow{2}{*}{$* *$} \\
\hline & Cont. & $\mathrm{N}=116$ & 29,84 & 5,77 & & & \\
\hline \multirow{2}{*}{ Razonamiento 4 (RZ 4) } & Exp. & $\mathrm{N}=249$ & 51,04 & 21,60 & \multirow{2}{*}{$-3,16$} & \multirow{2}{*}{, 11} & \\
\hline & Cont. & $\mathrm{N}=116$ & 54,75 & 19,60 & & & \\
\hline \multirow{2}{*}{ Memoria 4 (M 4) } & Exp. & $\mathrm{N}=248$ & 59,85 & 20,1 & \multirow{2}{*}{$-2,46$} & \multirow{2}{*}{, 014} & \multirow{2}{*}{$*$} \\
\hline & Cont. & $\mathrm{N}=116$ & 65,24 & 17,80 & & & \\
\hline \multirow{2}{*}{ R. Problema 6 (RP 6) } & Exp. & $\mathrm{N}=70$ & 7,29 & 3,58 & \multirow{2}{*}{,- 99} & \multirow{2}{*}{, 32} & \\
\hline & Cont. & $\mathrm{N}=24$ & 8,04 & 1,80 & & & \\
\hline
\end{tabular}

* significativa al 95\%; ** significativa al 99\%. 
TABLA 2 (continuación).

\begin{tabular}{|c|c|c|c|c|c|c|c|}
\hline Variables & \multicolumn{2}{|c|}{ Grupo } & Media & $\begin{array}{l}\text { Desv. } \\
\text { Tipic. }\end{array}$ & $\mathbf{T}$ & $\begin{array}{c}\text { Signf. } \\
\text { (bil) }\end{array}$ & Nivel \\
\hline \multirow{2}{*}{ Calculo 6 (CA 6) } & Exp. & $\mathrm{N}=70$ & 22,19 & 8,75 & \multirow{2}{*}{,- 727} & \multirow{2}{*}{,46 } & \\
\hline & Cont. & $\mathrm{N}=24$ & 23,54 & 4,32 & & & \\
\hline \multirow{2}{*}{ Razonamiento 6 (RZ 6) } & Exp. & $\mathrm{N}=71$ & 43,45 & 23,34 & \multirow{2}{*}{$-1,40$} & \multirow{2}{*}{, 16} & \\
\hline & Cont. & $\mathrm{N}=25$ & 50,60 & 16,98 & & & \\
\hline \multirow{2}{*}{ Memoria 6 (M 6) } & Exp. & $\mathrm{N}=71$ & 70,77 & 32,76 & \multirow{2}{*}{,377 } & \multirow{2}{*}{,70 } & \\
\hline & Cont. & $\mathrm{N}=25$ & 68,08 & 24,06 & & & \\
\hline \multirow{2}{*}{ CLEVPA I } & Exp. & $\mathrm{N}=172$ & 25,22 & 8,77 & \multirow{2}{*}{$-2,06$} & \multirow{2}{*}{, 03} & \multirow{2}{*}{$*$} \\
\hline & Cont. & $\mathrm{N}=72$ & 27,72 & 8,29 & & & \\
\hline \multirow{2}{*}{ CLEVPA II } & Exp. & $\mathrm{N}=160$ & 26,78 & 8,41 & \multirow{2}{*}{$-1,47$} & \multirow{2}{*}{,14 } & \\
\hline & Cont. & $\mathrm{N}=100$ & 28,30 & 7,64 & & & \\
\hline
\end{tabular}

* significativa al $95 \% ; * *$ significativa al $99 \%$.

A priori, no se hallan diferencias significativas en la variable C.L. ni en las variables de M; CA; R.P. y R.Z. de $3^{\circ}$ y $6^{\circ}$ de E.P., es decir, el G.E y el G.C. de estos niveles se muestran homogéneos; sin embargo, en $4^{\circ}$ y $5^{\circ}$ de E.P si se detecta esta diversidad. Existen diferencias significativas con una $\mathrm{p}<.01$ en RP y CA entre los alumnos del G.E. y G.C. de $4^{\circ}$ y $5^{\circ}$ de E.P. y en $M$; con una $p<.05$. En cuanto a la CLEVPA, señalar que sí se muestran diferencias significativas con una $\mathrm{p}<.05$ entre los alumnos del G.E. y G.C. en los primeros niveles, es decir en $3^{\circ}$ y $4^{\circ}$ de E.P. (CLEVPA I) pero no en el ciclo superior (CLEVPA II). Debido a que las diferencias entre ambos grupos ocurre en los niveles de $4^{\circ}$ y $5^{\circ}$ de E.P.; para tratar de precisar si las diferencias significativas que se aprecian en RP, CA y M se dan en un curso en concreto, es conveniente analizar cada nivel educativo por separado. Este mismo análisis también se realiza con el CLEVPA I para concretar si la diferencia que se aparece en el segundo ciclo de E.P. se repite al tomar cada nivel por separado ( $3^{\circ} \mathrm{y} 4^{\circ}$ de E.P.). A continuación, de nuevo se elabora un contraste de medias entre los alumnos de $4^{\circ}$ y $5^{\circ}$ de E.P. en las variables referidas a Resolución de Problemas, Cálculo y Memoria:

TABLA 3: Diferencias de medias en Resolución de Problemas entre el G.E. y el G.C. en $4^{\circ}$ y $5^{\circ}$ de E.P. antes del tratamiento.

\begin{tabular}{|c|c|c|c|c|c|c|c|c|}
\hline Variable & Curso & \multicolumn{2}{|c|}{ Grupo } & Media & Desv. Típ. & $T$ & Sig. (bil) & Nivel \\
\hline \multirow{4}{*}{$\begin{array}{l}\text { Resolución de } \\
\text { Problemas }\end{array}$} & \multirow{2}{*}{$4^{\circ}$ E.P. } & Exp. & 129 & 6,83 & 6,88 & \multirow{2}{*}{$-2,14$} & \multirow{2}{*}{, 00} & \multirow{2}{*}{$* *$} \\
\hline & & Cont. & 26 & 9,81 & 3,54 & & & \\
\hline & \multirow{2}{*}{$5^{\circ}$ E.P. } & Exp. & 118 & 8,84 & 4,08 & \multirow{2}{*}{$-2,86$} & \multirow{2}{*}{, 00} & \multirow{2}{*}{$* *$} \\
\hline & & Cont. & 89 & 11,02 & 6,82 & & & \\
\hline
\end{tabular}

* significativa al 95\%; ** significativa al $99 \%$. 
Se observa que en ambos niveles educativos sí existen diferencias significativas $(\mathrm{p}<.01)$ en la variable RP entre los grupos experimental y control a favor del G.C.

TABLA 4: Diferencias de medias en Cálculo entre el G.E. y el G.C. en $4^{\circ}$ y $5^{\circ}$ de E.P. antes del tratamiento.

\begin{tabular}{|c|c|c|c|c|c|c|c|c|}
\hline Variable & Curso & \multicolumn{2}{|c|}{ Grupo } & Media & Desv. Típ. & $T$ & Sig. (bil) & Nivel \\
\hline \multirow{4}{*}{ Cálculo } & \multirow{2}{*}{$4^{\circ}$ E.P. } & Exp. & 129 & 26,40 & 7,17 & \multirow{2}{*}{$-1,57$} & \multirow{2}{*}{, 03} & \multirow{2}{*}{$*$} \\
\hline & & Cont. & 26 & 29,50 & 4,94 & & & \\
\hline & \multirow{2}{*}{$5^{\circ}$ E.P. } & Exp. & 118 & 28,47 & 7,21 & \multirow{2}{*}{$-1,57$} & \multirow{2}{*}{,11 } & \\
\hline & & Cont. & 90 & 29,94 & 6,01 & & & \\
\hline
\end{tabular}

* significativa al 95\%; ** significativa al $99 \%$.

En la variable CA se detectan diferencias significativas entre el G.E. y G.C. en los alumnos de $4^{\circ}$ de E.P. con una $\mathrm{p}<.05$; nuevamente a favor del G.C; sin embargo, en el nivel educativo de $5^{\circ}$ de E.P. no se aprecian diferencias significativas.

TABLA 5: Diferencias de medias en Memoria entre el G.E. y el G.C. en $4^{\circ}$ y $5^{\circ}$ de E.P. antes del tratamiento.

\begin{tabular}{|c|c|c|c|c|c|c|c|c|}
\hline Variable & Curso & \multicolumn{2}{|c|}{ Grupo } & Media & Desv. Típ. & $T$ & Sig. (bil) & Nivel \\
\hline \multirow{4}{*}{ Memoria } & \multirow{2}{*}{$4^{\circ}$ E.P. } & Exp. & 129 & 55,60 & 19,34 & \multirow{2}{*}{$-4,68$} & \multirow{2}{*}{, 00} & \multirow{2}{*}{$* *$} \\
\hline & & Cont. & 26 & 74,50 & 15,64 & & & \\
\hline & \multirow{2}{*}{$5^{\circ}$ E.P. } & Exp. & 118 & 64,33 & 20,09 & \multirow{2}{*}{,662 } & \multirow{2}{*}{, 50} & \\
\hline & & Cont. & 90 & 62,57 & 17,57 & & & \\
\hline
\end{tabular}

* significativa al $95 \%$; ** significativa al $99 \%$.

Al igual que en la variable anterior, en la $\mathrm{M}$. se detectan diferencias significativas $(p<.01)$ entre el G.E. y el G.C. en $4^{\circ}$ de E.P.; pero no en el nivel educativo de $5^{\circ}$ E.P.

A continuación, se analizan los resultados obtenidos por los alumnos del $2^{\circ}$ ciclo de E.P. en la variable CLEVPA con objeto de analizar si podemos señalar diferencias significativas entre cada uno de los niveles que lo integran $\left(3^{\circ}\right.$ y $\left.4^{\circ}\right)$ :

TABLA 6: Diferencias de medias en Comprensión Lectora del Enunciado Verbal del Problema Aritmético entre el G.E. y el G.C. en $3^{\circ}$ y $4^{\circ}$ de E.P. antes del tratamiento.

\begin{tabular}{|c|c|l|r|r|r|c|c|c|}
\hline Variable & Curso & \multicolumn{2}{|c|}{ Grupo } & Media & Desv. Típ. & T & Sig. (bil) & Nivel \\
\hline \multirow{4}{*}{ CLEVPA I } & \multirow{2}{*}{$3^{\circ}$ E.P. } & Exp. & 49 & 26,76 & 6,61 & \multirow{2}{*}{, 44} & \multirow{2}{*}{, 72} & \\
\cline { 2 - 7 } & \multirow{2}{*}{$4^{\circ}$ E.P. } & Cont. & 46 & 26,22 & 8,03 & & & \\
\cline { 3 - 10 } & & Conp. & 123 & 24,60 & 9,46 & \multirow{2}{*}{$-3,20$} & \multirow{2}{*}{, 00} & $* *$ \\
\hline
\end{tabular}

* significativa al 95\%; ** significativa al 99\%. 
Las diferencias en el CLEVPA I, entre el G.E. y el G.C., no se dan por igual entre los niveles del segundo ciclo de E.P. De este modo, mientras sí se desprenden diferencias significativas entre los alumnos de $4^{\circ}$ E.P. (a favor del G.C.), éstas no se producen entre los alumnos de $3^{\circ}$ E.P.

Tras este primer análisis comparativo se observa que, en general, el G.E. y el G.C. se presentan como grupos homogéneos en las variables de estudio, excepto en $4^{\circ}$ de E.P donde sí se detectan diferencias significativas en las variables de RP, que (diferencia que se comparte con los alumnos de $5^{\circ}$ ), CA, M y CLEVPA a favor del GC.

Para continuar con el contraste de la hipótesis de la investigación, a continuación se comparan las puntuaciones medias obtenidas por el G.E. y el G.C. en cada una de las variables implicadas después del tratamiento efectuado. El análisis realizado consiste, en un contraste de medias mediante una prueba t para grupos independientes (G.E. y G.C.).

TABLA 7: Diferencia de medias en CL entre el GE y GC en cada curso después del tratamiento.

\begin{tabular}{|c|c|c|c|c|c|c|c|}
\hline Curso & Grupo & Media & $\mathbf{N}$ & Desv. & $\mathbf{T}$ & Sign. & Nivel \\
\hline \multirow{2}{*}{$3^{\circ}$} & Exp. & 16,20 & 25 & 4,113 & \multirow{2}{*}{,620 } & \multirow{2}{*}{,538 } & \\
\hline & Cont. & 15,46 & 26 & 4,384 & & & \\
\hline \multirow{2}{*}{$4^{\circ}$} & Exp. & 18,86 & 85 & 4,557 & \multirow{2}{*}{,973 } & \multirow{2}{*}{,332 } & \\
\hline & Cont. & 17,83 & 29 & 5,898 & & & \\
\hline \multirow{2}{*}{$5^{\circ}$} & Exp. & 23,00 & 74 & 5,480 & \multirow{2}{*}{3,191} & \multirow{2}{*}{, 00} & \multirow{2}{*}{$* *$} \\
\hline & Cont. & 19,72 & 76 & 6,983 & & & \\
\hline \multirow{2}{*}{$6^{\circ}$} & Exp. & 21,41 & 54 & 5,764 & \multirow{2}{*}{ 677 } & \multirow{2}{*}{, 50} & \\
\hline & Cont. & 20,48 & 25 & 5,425 & & & \\
\hline
\end{tabular}

* significativa al 95\%; ** significativa al 99\%.

El G.E. obtiene mejores puntuaciones medias en cada uno de los niveles educativos. Sin embargo, estas diferencias no se señalan como significativas excepto en $5^{\circ}$ de E.P., con una $\mathrm{p}<.01$.

En la tabla 8 se detectan diferencias significativas entre el G.E. y el G.C. en la CLEVPA, con una mejor puntuación media por parte del G.E. en el nivel de $3^{\circ}$ E.P., con una $p<.01$, y en $6^{\circ}$ E.P., con una $\mathrm{p}<.05$. Por otra parte, también detectamos diferencias significativas entre el G.E. y el G.C. pero a favor de éste último en los niveles de $4^{\circ}$ y $5^{\circ}$ de E.P. A este respecto, debemos recordar que ya detectamos diferencias significativas en el pretest con una puntuación media favorable al G.C., por lo que se tendrá en cuenta en análisis posteriores para controlar la influencia que esto pudiera tener sobre la CLEVPA.

En RP (Tabla 9) se observa que tanto el G.E. de $3^{\circ}$ de E.P. como el de $6^{\circ}$ de E.P. arrojan diferencias significativas, con una $\mathrm{p}<.05$ y $\mathrm{p}<.01$ respectivamente, al comparar su rendimiento con el G.C. En ambos casos, el G.E. obtiene una puntuación mayor que su respectivo G.C. Sin embargo, en los alumnos de $4^{\circ}$ y $5^{\circ}$ de E.P. sucede que los alumnos 
del G.C. han obtenido una mejor puntuación que los del G.E., llegando a ser significativa $(\mathrm{p}<.05)$ en el nivel de $4^{\circ}$. En análisis posteriores, se tendrán en cuenta las diferencias pretest detectadas en RP entre el G.E, y el G.C., con puntuaciones medias favorables a este último grupo.

TABLA 8: Diferencia de medias en Comprensión Lectora del Enunciado Verbal del Problema Aritmético entre el GE y GC en cada curso después del tratamiento.

\begin{tabular}{|c|c|c|c|c|c|c|c|}
\hline Curso & Grupo & Media & $\mathbf{N}$ & Desv. & $\mathbf{T}$ & Sign. & Nivel \\
\hline \multirow{2}{*}{$3^{\circ}$} & Exp. & 66,88 & 49 & 16,52 & \multirow{2}{*}{3,61} & \multirow{2}{*}{,00 } & \multirow{2}{*}{$* *$} \\
\hline & Cont. & 65,54 & 46 & 20,08 & & & \\
\hline \multirow{2}{*}{$4^{\circ}$} & Exp. & 61,50 & 123 & 23,64 & \multirow{2}{*}{$-3,16$} & \multirow{2}{*}{00} & \multirow{2}{*}{$* *$} \\
\hline & Cont. & 75,96 & 26 & 20,56 & & & \\
\hline \multirow{2}{*}{$5^{\circ}$} & Exp. & 61,88 & 106 & 20,54 & \multirow{2}{*}{$-3,39$} & \multirow{2}{*}{, 00} & \multirow{2}{*}{$* *$} \\
\hline & Cont. & 72,13 & 75 & 19,16 & & & \\
\hline \multirow{2}{*}{$6^{\circ}$} & Exp. & 76,85 & 54 & 18,45 & \multirow{2}{*}{2,28} & \multirow{2}{*}{,02 } & \multirow{2}{*}{$*$} \\
\hline & Cont. & 66,60 & 25 & 18,73 & & & \\
\hline
\end{tabular}

* significativa al 95\%; ** significativa al $99 \%$.

TABLA 9: Diferencia de medias en Resolución de Problemas entre el GE y GC en cada curso después del tratamiento.

\begin{tabular}{|c|c|c|c|c|c|c|c|}
\hline Curso & Grupo & Media & $\mathbf{N}$ & Desv. & $\mathbf{T}$ & Sign. & Nivel \\
\hline \multirow{2}{*}{$3^{\circ}$} & Exp. & 12,19 & 37 & 1,86 & \multirow{2}{*}{3,02} & \multirow{2}{*}{, 00} & \multirow{2}{*}{$*$} \\
\hline & Cont. & 10,20 & 50 & 3,65 & & & \\
\hline \multirow{2}{*}{$4^{\circ}$} & Exp. & 8,81 & 91 & 4,11 & \multirow{2}{*}{$-2,02$} & \multirow{2}{*}{, 04} & \multirow{2}{*}{$*$} \\
\hline & Cont. & 10,46 & 28 & 2,36 & & & \\
\hline \multirow{2}{*}{$5^{\circ}$} & Exp. & 13,15 & 67 & 12,45 & \multirow{2}{*}{,98 } & \multirow{2}{*}{, 32} & \\
\hline & Cont. & 11,51 & 73 & 6,50 & & & \\
\hline \multirow{2}{*}{$6^{\circ}$} & Exp. & 9,84 & 50 & 2,40 & \multirow{2}{*}{4,60} & \multirow{2}{*}{, 00} & \multirow{2}{*}{$* *$} \\
\hline & Cont. & 6,80 & 25 & 3,21 & & & \\
\hline
\end{tabular}

* significativa al 95\%; ** significativa al 99\%.

Los alumnos del G.E. de $3^{\circ}$ de E.P. obtienen una puntuación significativamente superior $(\mathrm{p}<.05)$ que la de sus compañeros del G.C. (Tabla 10). Esta situación también se produce entre los alumnos de $4^{\circ}$ E.P., pero con una $\mathrm{p}<.01$. Por otra parte, en $5^{\circ}$ y en $6^{\circ}$ de E.P., los alumnos del G.E. también obtienen una mejor puntuación en comparación con el G.C., aunque estas diferencias no se señalan como significativas. 
TABLA 10: Diferencia de medias en RZ entre el GE y GC en cada curso después del tratamiento.

\begin{tabular}{|c|c|c|c|c|c|c|c|}
\hline Curso & Grupo & Media & $\mathbf{N}$ & Desv. & $\mathbf{T}$ & Sign. & Nivel \\
\hline \multirow{2}{*}{$3^{\circ}$} & Exp. & 77,71 & 38 & 14,97 & \multirow{2}{*}{2,01} & \multirow{2}{*}{, 04} & \multirow{2}{*}{$*$} \\
\hline & Cont. & 70,70 & 50 & 16,99 & & & \\
\hline \multirow{2}{*}{$4^{\circ}$} & Exp. & 65,74 & 107 & 22,27 & \multirow{2}{*}{$-3,10$} & \multirow{2}{*}{, 00} & \multirow{2}{*}{$* *$} \\
\hline & Cont. & 51,50 & 27 & 16,50 & & & \\
\hline \multirow{2}{*}{$5^{\circ}$} & Exp. & 64,48 & 67 & 14,67 & \multirow{2}{*}{1,88} & \multirow{2}{*}{, 05} & \\
\hline & Cont. & 58,78 & 82 & 20,86 & & & \\
\hline \multirow{2}{*}{$6^{\circ}$} & Exp. & 59,32 & 52 & 22,61 & \multirow{2}{*}{1,69} & \multirow{2}{*}{,09 } & \\
\hline & Cont. & 50,21 & 25 & 21,07 & & & \\
\hline
\end{tabular}

* significativa al $95 \%$; ** significativa al $99 \%$.

En la tabla 11 se detecta que, después del entrenamiento en estrategias, en los alumnos de $6^{\circ}$ E.P. del G.E. y el G.C. se detectan diferencias significativas, con una $\mathrm{p}<.01$, a favor del G.E. Los alumnos de $3^{\circ}$ y $5^{\circ}$ E.P. del G.E. obtienen mejores resultados que sus compañeros del G.C., sin embargo, estas diferencias no son significativas. En $4^{\circ}$ E.P. tampoco supone una diferencia significativa el ligero ascenso del G.C. frente al G.E, a pesar de que en la situación pretest sí detectamos diferencias significativas a favor del G.C. en esta variable.

TABLA 11: Diferencia de medias en CA entre el GE y GC en cada curso después del tratamiento.

\begin{tabular}{|c|c|c|c|c|c|c|c|}
\hline Curso & Grupo & Media & $\mathbf{N}$ & Desv. & $\mathbf{T}$ & Sign. & Nivel \\
\hline \multirow{2}{*}{$3^{\circ}$} & Exp. & 46,95 & 37 & 7,47 & \multirow{2}{*}{,60 } & \multirow{2}{*}{, 54} & \\
\hline & Cont. & 45,82 & 49 & 9,26 & & & \\
\hline \multirow{2}{*}{$4^{\circ}$} & Exp. & 29,02 & 87 & 9,79 & \multirow{2}{*}{,- 75} & \multirow{2}{*}{,45 } & \\
\hline & Cont. & 30,46 & 28 & 4,02 & & & \\
\hline \multirow{2}{*}{$5^{\circ}$} & Exp. & 31,61 & 67 & 6,68 & \multirow{2}{*}{1,06} & \multirow{2}{*}{, 28} & \\
\hline & Cont. & 30,47 & 81 & 6,35 & & & \\
\hline \multirow{2}{*}{$6^{\circ}$} & Exp. & 27,22 & 50 & 5,00 & \multirow{2}{*}{3,00} & \multirow{2}{*}{, 00} & \multirow{2}{*}{$* *$} \\
\hline & Cont. & 22,48 & 25 & 8,66 & & & \\
\hline
\end{tabular}

* significativa al $95 \%$; ** significativa al $99 \%$.

Por último se observa en la tabla 12 que existen diferencias significativas en la variable M. al comparar los resultados del G.E. y del G.C. tras el entrenamiento. Sin embargo, debemos precisar que tanto los alumnos de $3^{\circ}$ de E.P. del G.E. como los de $6^{\circ}$ de E.P. obtienen unas puntuaciones medias superiores a las obtenidas por el G.C; con una $p<.05$ en $3^{\circ}$ 
de E.P. y con una $\mathrm{p}<.01$ en $6^{\circ}$ de E.P. También se detectan diferencias significativas $(p<.05)$ en $4^{\circ}$ E.P. y en $5^{\circ}$ E.P. $(p<.01)$ pero, y a diferencia de los anteriores niveles, a favor del G.C.

TABLA 12: Diferencia de medias en Memoria entre el GE y GC en cada curso.

\begin{tabular}{|c|c|c|c|c|c|c|c|}
\hline Curso & Grupo & Media & $\mathbf{N}$ & Desv. & $\mathbf{T}$ & Sign. & Nivel \\
\hline \multirow{2}{*}{$3^{\circ}$} & Exp. & 69,68 & 38 & 13,06 & \multirow{2}{*}{2,59} & \multirow{2}{*}{, 01} & \multirow{2}{*}{$*$} \\
\hline & Cont. & 61,78 & 51 & 14,99 & & & \\
\hline \multirow{2}{*}{$4^{\mathrm{o}}$} & Exp. & 67,49 & 85 & 18,22 & \multirow{2}{*}{$-2,40$} & \multirow{2}{*}{, 01} & \multirow{2}{*}{$*$} \\
\hline & Cont. & 76,89 & 28 & 17,12 & & & \\
\hline \multirow{2}{*}{$5^{\circ}$} & Exp. & 68,30 & 66 & 17,44 & \multirow{2}{*}{$-3,10$} & \multirow{2}{*}{, 00} & \multirow{2}{*}{$* *$} \\
\hline & Cont. & 77,23 & 82 & 17,33 & & & \\
\hline \multirow{2}{*}{$6^{\circ}$} & Exp. & 94,98 & 52 & 18,53 & \multirow{2}{*}{$\begin{array}{l}6,85 \\
7,69\end{array}$} & \multirow{2}{*}{, 00} & \multirow{2}{*}{$* *$} \\
\hline & Cont. & 66,56 & 25 & 13,26 & & & \\
\hline
\end{tabular}

* significativa al $95 \%$; ** significativa al $99 \%$.

A lo largo de estos análisis se observa que tras el periodo de entrenamiento se han detectado diferencias significativas "esperadas", esto es, a favor del G.E., y diferencias significativas "no esperadas", es decir, a favor del G.C. en concreto en los niveles de $4^{\circ}$ y $5^{\circ}$ de E.P. Por este motivo, resulta oportuno controlar aquellas diferencias que se detectaron antes del entrenamiento debido a la posible incidencia que pudieran tener sobre la CLEVPA. Con este objetivo, a continuación se lleva a cabo un Análisis de Covarianza (ANCOVA).

En primer lugar, se realiza un Análisis de Covarianza (ANCOVA) tomando como V.D. la CLEVPA y como covariable el CA en el nivel educativo de $4^{\circ}$ de E.P.

TABLA 13: ANCOVA en CLEVPA I tomando como covariable el CA en $4^{\circ}$ E.P. Variable dependiente: clepa post.

\begin{tabular}{|l|c|r|r|r|c|}
\hline \multicolumn{1}{|c|}{ Fuente } & $\begin{array}{c}\text { Suma de } \\
\text { cuadrados tipo III }\end{array}$ & \multicolumn{1}{c|}{ gl } & $\begin{array}{c}\text { Media } \\
\text { cuadrática }\end{array}$ & F & Significación \\
\hline Modelo corregido & $8.879,030^{*}$ & 2 & $4.439,515$ & 8,899 &, 000 \\
\hline Intersección & $15.783,309$ & 1 & $15.783,309$ & 31,637 &, 000 \\
\hline CALPREZ & $4.918,240$ & 1 & $4.918,240$ & 9,858 &, 002 \\
\hline GRUPO & $2.488,225$ & 1 & $2.488,225$ & 4,988 &, 027 \\
\hline Error & $69.344,913$ & 139 & 498,884 & & \\
\hline Total & $665.887,500$ & 142 & & & \\
\hline Total corregida & $78.223,944$ & 141 & & & \\
\hline
\end{tabular}

$* \mathrm{R}$ cuadrado $=, 114(\mathrm{R}$ cuadrado corregida $=, 101)$. 
Se evidencian diferencias significativas (.027) con una $\mathrm{p}<.05$, entre el G.E. y el G.C. en la CLEVPA aun teniendo en cuenta las diferencias previas detectadas en CA. Esto significa que el G.C. obtiene un rendimiento significativamente mejor que el GE incluso controlando las diferencias previas detectadas en CA.

A continuación, realizamos un nuevo Análisis de Covarianza tomando como V.D. la CLEVPA y como covariable la RP para el nivel de $4^{\circ}$ de E.P.

TABLA 14: ANCOVA en CLEVPA I tomando como covariable la RP en $4^{\circ}$ E.P. Variable dependiente: clepa post.

\begin{tabular}{|l|c|r|r|r|r|}
\hline \multicolumn{1}{|c|}{ Fuente } & $\begin{array}{c}\text { Suma de } \\
\text { cuadrados tipo III }\end{array}$ & gl & $\begin{array}{c}\text { Media } \\
\text { cuadrática }\end{array}$ & F & Significación \\
\hline Modelo corregido & $27.403,739^{*}$ & 2 & $13.701,870$ & 41,632 &, 000 \\
\hline Intersección & $24.781,112$ & 1 & $24.781,112$ & 75,295 &, 000 \\
\hline RPPREZ & $24.533,594$ & 1 & $24.533,594$ & 74,543 &, 000 \\
\hline GRUPO & 1,359 & 1 & 1,359 &, 004 &, 949 \\
\hline Error & $43.114,731$ & 131 & 329,120 & & \\
\hline Total & $645.150,000$ & 134 & & & \\
\hline Total corregida & $70.518,470$ & 133 & & & \\
\hline
\end{tabular}

$* \mathrm{R}$ cuadrado $=, 389(\mathrm{R}$ cuadrado corregida $=, 379)$.

No se detectan diferencias significativas entre el G.E. y el G.C. en la CLEVPA tras controlar las diferencias que se detectaban entre ambos grupos en la RP antes del tratamiento.

De nuevo se realiza un Análisis de Covarianza tomando como covariable la $\mathrm{M}$. en $4^{\circ}$ de E.P.:

TABLA 15: ANCOVA en CLEVPA I tomando como covariable la $M$ en $4^{\circ}$ E.P. Variable dependiente: clepa post.

\begin{tabular}{|l|r|r|r|r|c|}
\hline \multicolumn{1}{|c|}{ Fuente } & \multicolumn{1}{c|}{$\begin{array}{c}\text { Suma de } \\
\text { cuadrados tipo III }\end{array}$} & \multicolumn{1}{c|}{ gl } & $\begin{array}{c}\text { Media } \\
\text { cuadrática }\end{array}$ & \multicolumn{1}{c|}{ F } & Significación \\
\hline Modelo corregido & $4.875,051^{*}$ & 2 & $2.437,526$ & 5,431 &, 005 \\
\hline Intersección & $36.518,819$ & 1 & $36.518,819$ & 81,369 &, 000 \\
\hline MEMPREZ & $3.074,919$ & 1 & $3.074,919$ & 6,851 &, 009 \\
\hline GRUPO & 648,329 & 1 & 648,329 & 1,445 &, 231 \\
\hline Error & $104.122,289$ & 232 & 448,803 & & \\
\hline Total & $1.098 .625,000$ & 235 & & & \\
\hline Total corregida & $108.997,340$ & 234 & & & \\
\hline
\end{tabular}

$* \mathrm{R}$ cuadrado $=, 045(\mathrm{R}$ cuadrado corregida $=, 036)$. 
En este caso tampoco se detectan diferencias significativas entre el G.E. y el G.C., en la Comprensión Lectora del Enunciado Verbal del Problema Aritmético controlando la incidencia previa de la Memoria en $4^{\circ}$ de E.P.

Finalmente, se realiza un último Análisis de Covarianza en el nivel educativo de $5^{\circ}$ de E.P. adoptando de nuevo como V.D. la CLEVPA y, como covariable, la RP:

En la tabla 16 se observa que existen diferencias significativas $(0,00)$ con una $p<.01$ entre el G.E. y el G.C. en la CLEVPA incluso considerando las diferencias significativas que se establecían entre ambos grupos antes del tratamiento en RP.

TABLA 16: ANCOVA en CLEVPA II tomando como covariable la RP en $5^{\circ}$ E.P. Variable dependiente: clepa post.

\begin{tabular}{|l|c|r|r|r|c|}
\hline \multicolumn{1}{|c|}{ Fuente } & $\begin{array}{c}\text { Suma de } \\
\text { cuadrados tipo III }\end{array}$ & \multicolumn{1}{c|}{ gl } & $\begin{array}{c}\text { Media } \\
\text { cuadrática }\end{array}$ & \multicolumn{1}{c|}{ F } & Significación \\
\hline Modelo corregido & $13.162,016^{*}$ & 2 & $6.581,008$ & 19,004 &, 000 \\
\hline Intersección & $35.883,145$ & 1 & $35.883,145$ & 103,620 &, 000 \\
\hline RPPRE & $7.758,625$ & 1 & $7.758,625$ & 22,405 &, 000 \\
\hline GRUPO & $2.633,175$ & 1 & $2.633,175$ & 7,604 &, 007 \\
\hline Error & $54.714,537$ & 158 & 346,295 & & \\
\hline Total & $751.681,250$ & 161 & & & \\
\hline Total corregida & $67.876,553$ & 160 & & & \\
\hline
\end{tabular}

$* \mathrm{R}$ cuadrado $=, 194(\mathrm{R}$ cuadrado corregida $=, 184)$.

\section{Conclusiones e implicaciones}

A partir de los datos obtenidos confirmamos que el entrenamiento en estrategias comprensivas y metacomprensivas seleccionadas a partir del Programa Comprender y Aprender ha beneficiado significativamente tanto la comprensión lectora del problema aritmético de enunciado verbal de los alumnos sometidos a dicho entrenamiento como otras variables relacionadas con este proceso, sin embargo, este beneficio no ha sido homogéneo entre los diferentes niveles educativos.

Los niveles educativos que más se han beneficiado del entrenamiento en estrategias de comprensión y metacomprensión son $3^{\circ}$ y $6^{\circ}$ E.P.; detectándose diferencias significativas en la Comprensión Lectora del Enunciado Verbal, así como en la Resolución de Problemas y en Memoria. Por otra parte, mientras en $3^{\circ}$ también se detectan diferencias significas en Razonamiento éstas no se dan en $6^{\circ}$ E.P; en cambio, en este nivel sí se producen otras diferencias significativas como las detectadas en Cálculo.

Los resultados obtenidos en los niveles de $4^{\circ}$ y $5^{\circ}$ de E.P. después del entrenamiento no han sido tan homogéneos ni esperados como los acontecidos en los cursos anteriores, principalmente como consecuencia de las diferencias detectadas antes del entrenamiento a favor del G.C. Estas diferencias de partida pueden estar motivadas por las dificultades encontradas para realizar una estricta aleatorización de la muestra. 
En $4^{\circ}$ de E.P., controlando la incidencia de las diferencias de partida detectadas en el Cálculo a favor del G.C., se obtiene que el entrenamiento ha beneficiado la Comprensión Lectora del Enunciado Verbal del Problema Aritmético del G.E., sin embargo, esta mejoría no parece producirse al controlar otras diferencias de partida detectadas en Resolución de Problemas y en Memoria. En este nivel también se producen diferencias significativas a favor del G.E. en Razonamiento.

El curso de $5^{\circ}$ E.P. es el único en el que se detectan diferencias significativas en la Comprensión Lectora a favor del G.E. Por otra parte, dado que en este nivel también se detectaron diferencias significativas antes del entrenamiento en la Resolución de Problemas en el G.C., el control de la incidencia de dicha variable nos permite confirmar la existencia de diferencias significativas en la Comprensión Lectora del Enunciado del Problema, de hecho, aquella diferencia significativa en Resolución de Problemas se convierte en no significativa después del entrenamiento en estrategias.

De lo expuesto en este trabajo y a la luz de los resultados obtenidos en esta investigación, queremos resaltar que el objetivo último del uso de estrategias de comprensión lectora en el enunciado matemático es facilitar la construcción de un modelo mental flexible y cambiante conforme el alumno es capaz de interpretarlo, en palabras de Kintsch y Greeno (1985: 101): "un proceso dinámico de cambio de la representación, gracias al cual el alumno pasa de una representación inadecuada, en la que atribuye a la tarea propiedades que no tiene, a una representación adecuada, y de una representación incompleta a otra completa".

Las implicaciones educativas que se extraen de este trabajo hacen referencia a la mejora del proceso de enseñanza de la Resolución de Problemas, empezando por entrenar a los alumnos en estrategias comprensivas y metacomprensivas para la mejora de la comprensión lectora del enunciado del problema aritmético. En este sentido, proponemos algunas indicaciones como:

- Entrenar a los alumnos en la comprensión lectora del enunciado por medio de la aplicación de estrategias comprensivas y metacomprensivas (se proponen las empleadas en este trabajo) a partir de las que ellos manejan, mediante actividades y textos que requieren el uso de dichas estrategias, con el fin de que el alumno experimente su funcionalidad.

- Emplear las estrategias comprensivas y metacomprensivas como un recurso metodológico en el proceso de enseñanza y aprendizaje por medio de los textos escolares de forma transversal.

Es incuestionable que, en la medida en que se facilite al alumno estrategias para leer correctamente los enunciados de los problemas le estaremos ayudando, primero, a que la lectura del enunciado no se convierta en sí misma en una dificultad o "problema" añadido que solventar y, segundo, le estaremos facilitando el procedimiento básico por antonomasia para acceder al conocimiento matemático, esto es, el proceso de resolución de problemas.

\section{Referencias bibliográficas}

Anderson, J. R. (1993). Problem solving and learning. American Psychologist, 48, 35-44.

Anderson, J. R. (1984). Role of the reader's schema in comprehension, learning, and memory. En R. C. Anderson, J. Osborn y R. J. Tierney (Eds.), Learning to read in American schools: Basal readers and content texts. Hillsdale, N.J.: L.E.A. 
Ballester, L. (2001). Bases metodológicas de la investigación educativa. Palma: Universidad de las Islas Baleares. Colección de materiales didácticos, $\mathrm{n}^{\circ} 086$.

Bransford, J. D. y Stein, B. S. (1993). Solución IDEAL de Problemas. Guía para mejor pensar, aprender y crear. Barcelona: Labor. (Traducción de 1988, The IDEAL problem solver: A guide for improving thinking, learning and creativity. New York: Freemen).

Carr, M. y Hettinger, H. (2003). Perspectives on Mathematics Strategy Development. En J. M. Royer (Ed.), Mathematical Cognition. Greenwich, Connecticut: Information Age Publishing.

Castro Martínez, E. (1995). Niveles de comprensión en problemas verbales de comparación multiplicativa. Granada: COMERAS, Colección Mathema.

Chamoro, $M^{\mathrm{a}}$. C. (2003). Las dificultades de lectura y comprensión de los problemas matemáticos escolares. UNO. Revista de Didáctica de las matemáticas, 33, 99-119.

Fernández Bravo, J. A. (2000). Técnicas creativas para la resolución de problemas matemáticos. Barcelona: Cisspraxis

Geary, D. y Orad, M. K (2003). Learning Disabilities in Basic Mathematics: Deficits in Memory and Cognition. En J. M. Royer (Ed.), Mathematical Cognition. Greenwich, Connecticut: Information Age Publishig.

Kintsch, W. y Greeno, J. G. (1985). Understanding and Solving Word Arithmetic Problems. Psychological Review, 92, 1, 109-129.

Mason, J.; Burton, L. y Stacey, K. (1989). Pensar matemáticamente. Barcelona: Labor-MEC.

Maza, C. (1995). Aritmética y representación. De la comprensión del texto al uso de los materiales. Barcelona: Piados.

Mayer, R. E. (2003). Mathematical Problem Solving. En J. M. Royer (Ed.), Mathematical Cognition. Greenwich, Connecticut: Information Age Publishig.

Mayer, R. E. (1992). Guiding studen's cognitive processing of scientific information in text. En M. Pressley, K. R. Harris y J. T. Guthrie (eds.): Promoting academic competence and literacy in school. San Diego: Academic Press.

Mayer, R. E. (1986a). Pensamiento, resolución de problemas y cognición. Barcelona: Paidós.

Mayer, R. E. (1986b). Capacidad matemática. En R. J. Sternberg (Ed.), Las capacidades humanas. Barcelona: Labor.

Miranda, A.; Fortes, C. y Gil D. (2000). Dificultades del aprendizaje de las matemáticas. Un enfoque evolutivo. Málaga: Aljibe.

Pérez Echeverría, M. P. (1994). La solución de problemas en matemáticas. En J. I. Pozo y otros (Coord.), La solución de problemas. Madrid: Santillana.

Pérez Echeverría, M. P. y Pozo, J. I. (1994). Aprender a resolver problemas y resolver problemas para aprender. En J. I. Pozo y otros (Coord.), La solución de problemas. Madrid: Santillana.

Polya, G. (1990). Cómo plantear y resolver problemas. México: Trillas. (1ª edición 1965).

Pozo, J. I. y Postigo, Y. (1993). Las estrategias de aprendizaje como contenido del currículo. En C. Monereo (Comp.), Las estrategias de aprendizaje: Procesos, contenidos e interacción. Barcelona: Ediciones Doménech.

Repetto, E.; Sutil, I.; Manzano, N. y cols. (Beltrán, S. G. y Téllez, J. A.). (2001a). Programa Comprender y Aprender en el Aula. Manual del Profesor. Madrid: UNED.

Schoenfeld, H. A. (1992). Learning to think mathematically: problem solving, metacognition and sense making in mathematics. En D. A. Grouws (Ed.), Handbook of research on mathematics teaching and learning. Nueva York: McMillan Publishing Company. 
Schoenfeld, A. H. (1985). Metacognitive and Epistemological Issues in Mathematical Understanding. En E. A. Silver (Ed.), Teaching and Learning Mathematicl Problem Solving: Multiple Research Perspectives. Hillsdale, NJ: Lawrence Erlbaum Associates.

Verschaffel, L.; Greer, B. y De Corte, E. (2000). Making Sense of word problems. Lisse: Swets \& Zeitlinger.

Fecha de recepción: 29-11-04

Fecha de revisión: 09-01-06

Fecha de aceptación: 25-04-06 